\title{
High Resolution Soft X-ray Tomography of Large Samples by Focal Series Projections
}

\author{
Joaquín Otón ${ }^{1}$, Eva Pereiro ${ }^{2}$, José Javier Conesa ${ }^{2}$, Francisco Javier Chichón ${ }^{1}$, José L. Carrascosa ${ }^{3}$ and \\ José María Carazo ${ }^{1}$ \\ 1. Structure of Macromolecules, Centro Nacional de Biotecnología CSIC, Madrid, SPAIN. \\ 2. Experiment Division, ALBA Light Source, Cerdanyola del Vallès, SPAIN. \\ 3. Unidad Asociada CNB, Instituto Madrileño de Estudios Avanzados en Nanociencia (IMDEA \\ Nanociencia), Madrid, SPAIN.
}

Soft X-ray tomography (SXT) has become one of the most recent structural biology techniques for imaging biological samples. It allows the exploration of the internal structure by imaging whole cells when preserved in cryo-conditions without use of staining agents in the so-called water window energy range (between 284 and $543 \mathrm{eV}$ ). Projections are imaged by Fresnel zone plate (FZP) lenses with spatial resolution in the few tens of nanometers with a field of view in the range 10-20 x 10-20 $\mathrm{\mu m}^{2}$. Information from these projections is then combined by tomographic techniques to generate threedimensional (3D) maps of the specimen. However, due to the intrinsic limited depth of field (DOF) of the microscope objective lens, SXT is far from currently being delivering its maximum capability in terms of isotropic volume resolution. Indeed, the limited DOF produces images where each plane of the sample contributes with a different transfer function, leading to 3D maps where certain parts of the specimen are blurred [1]. This involves not only thick samples but large samples, as details placed in lateral positions of the field of view at $0^{\circ}$ tilt angle will be shifted to different z-planes when the sample is tilted.

After analyzing the image formation process in detail, we provide XTEND, a new mathematical approach that allows for the quantitative SXT blurring inversion using focal series projections, that is, images acquired at different defocus (Figure 1). Following these ideas, the SXT microscope Mistral at the Spanish synchrotron ALBA has been modified so as to automatically acquire the necessary additional information in a fast and reliable manner to specifically calculate the optimal XTEND parameters at each tilt angle considering field of view and sample thickness.

\section{References:}

[1] J. Otón et al, J. Struct Biol, 178 (1) (2012), p. 29. 

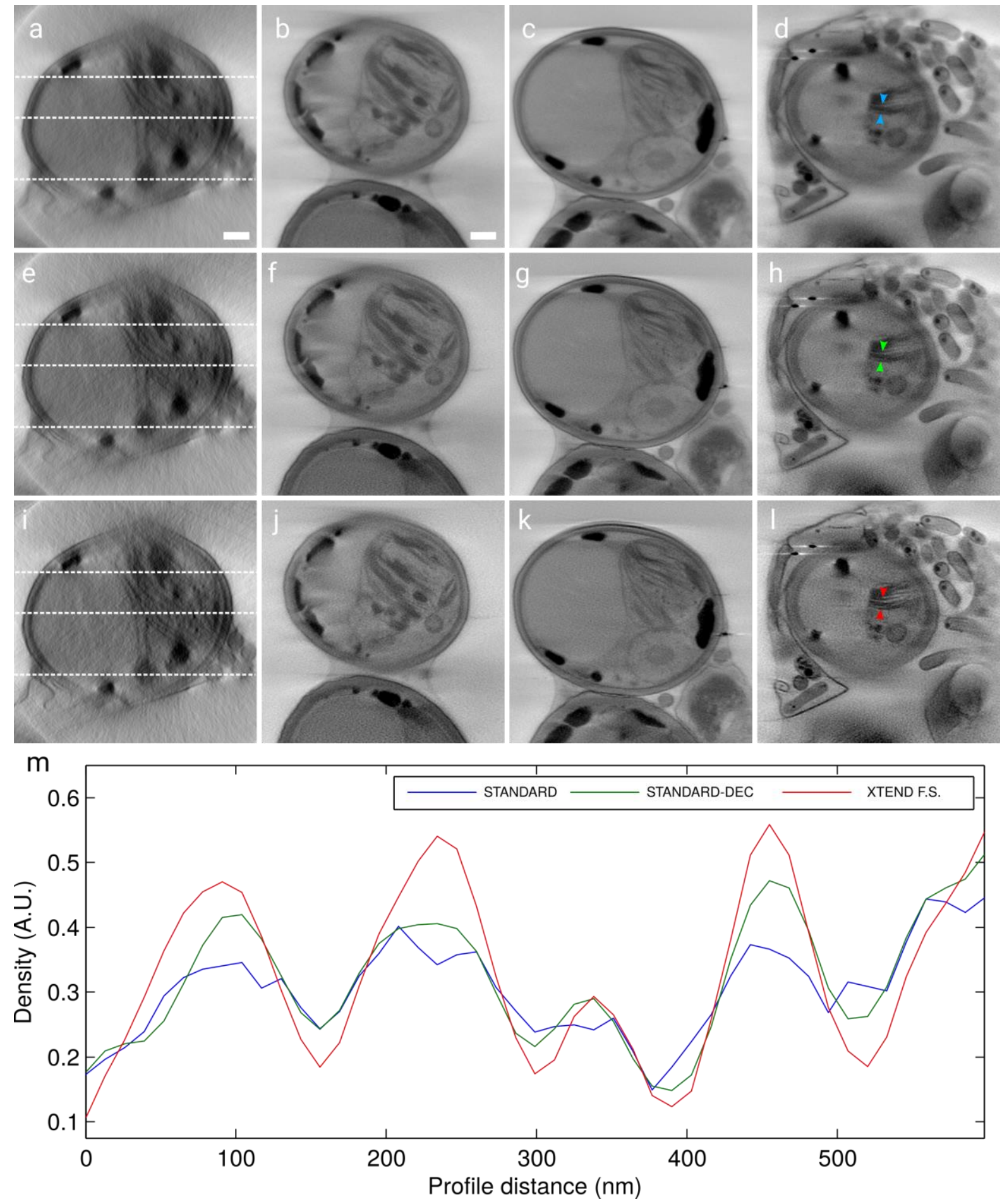

Figure 1. Comparison of the different collection methods on a Scenedemus cell experimental tomography imaged by a $40 \mathrm{~nm}$ FZP in Mistral microscope (61.9 nm resolution, $3.3 \mu \mathrm{m}$ DOF): standard tomography (first row), deconvolved standard tomography (second) and XTEND (third) reconstructions; (a, e, i) x-z planes where x-y slices at position -1.6 $\mu \mathrm{m}(\mathrm{b}, \mathrm{f}, \mathrm{j}), 0 \mu \mathrm{m}(\mathrm{c}, \mathrm{g}, \mathrm{k})$ and $2.4 \mu \mathrm{m}(\mathrm{d}, \mathrm{h}, \mathrm{l})$ are marked. Scale bars $=1 \mu \mathrm{m}$. (m) Density profiles along the paths pointed between color markers (standard, deconvolved standard and XTEND in blue, green and red, respectively) in slices (d, h, l). 